http://jmscr.igmpublication.org/home/ ISSN (e)-2347-176x ISSN (p) 2455-0450

crossref DOI: https://dx.doi.org/10.18535/jmscr/v9i9.11

\title{
Early Covid-19 disease is driven by replication of virus and Late disease is driven by a dysregulated immune/inflammatory response
}

\author{
Author \\ Dr Rajendra Tatu Nanavare \\ Chest Physician \& Ex. MS GTB Hospitals Sewri Mumbai 15
}

\section{Introduction}

The COVID-19 pandemic is perhaps the greatest healthcare challenge of a generation. It is a new pathogen that is highly contagious, can spread quickly, and must be considered capable of causing enormous health, economic and societal impacts in any setting. COVID-19 is caused by the most recently discovered coronavirus. This new virus and the disease were unknown before the outbreak began in Wuhan, China, in December 2019. COVID-19 has now affected several countries and has caused widespread death. ${ }^{(1)}$

The World Health Organization declared COVID19 as a global pandemic on March 11, 2020. The disease primarily spreads via close contact of respiratory droplets generated by infected individuals. So early detection and isolation of cases is the key to control epidemic by the time vaccine or anti-viral becomes available. WHO sets its mission for COVID control as "detect, protect and treat" to break the chain of transmission of SARSCoV-2. ${ }^{(2)}$

The world is amidst a pandemic that is presenting one of the greatest public health challenges of the twenty-first century. A new coronavirus has jumped the interspecies barrier, and it possesses the capability of efficient person-to-person transmission and selectively kills older people and those with underlying chronic conditions. ${ }^{(3)}$

Patients with SARS-CoV-2 infection can experience a range of clinical manifestations, from no symptoms to critical illness. In general, adults with SARS-CoV-2 infection can be grouped into the following severity of illness categories. However, the criteria for each category may overlap or vary across clinical guidelines and clinical trials, and a patient's clinical status may change over time.

Asymptomatic or Presymptomatic Infection: Individuals who test positive for SARS-CoV-2 using a virologic test (i.e., a nucleic acid amplification test [NAAT] or an antigen test) but who have no symptoms that are consistent with COVID-19.

Mild Illness: Individuals who have any of the various signs and symptoms of COVID-19 (e.g., fever, cough, sore throat, malaise, headache, muscle pain, nausea, vomiting, diarrhea, loss of taste and smell) but who do not have shortness of breath, dyspnea, or abnormal chest imaging.

Moderate Illness: Individuals who show evidence of lower respiratory disease during clinical assessment or imaging and who have an oxygen saturation $(\mathrm{SpO} 2) \geq 94 \%$ on room air at sea level. 
Severe Illness: Individuals who have $\mathrm{SpO} 2<94 \%$ on room air at sea level, a ratio of arterial partial pressure of oxygen to fraction of inspired oxygen $(\mathrm{PaO} 2 / \mathrm{FiO} 2)<300 \mathrm{~mm} \mathrm{Hg}$, respiratory frequency $>30$ breaths/min, or lung infiltrates $>50 \%$.

Critical Illness: Individuals who have respiratory failure, septic shock, and/or multiple organ dysfunction Two main processes are thought to drive the pathogenesis of COVID-19. ${ }^{(4)}$

\section{Case History}

Patient male 35 yrs old electrician, Food habit Vegetarian, non-Alcoholic, non-smoker, no major illness in past, h/o Covishield vaccine 1 st dose taken 14 days back. and H/o HTN 1 yr and on anti HTN.

First day Develop High grade fever with chills, went to General practitioner in evening IV fluids and tablets given by him, Second and third day taken rest at home. Fourth day attended office and again got fever high grade 104F with chills. Meet me and I have started Antibiotics Azithromycin (500mg OD), and doxycycline (100mg BD) with symptomatic treatment. and advise for CBC, CRP, Dengue test, Malarial test with Urine and x-ray chest, fifth day having fever $100.2 \mathrm{~F}$ and loose motion started. Report came RT PCR, Dengue, Malarial Negative, Urine was having occult blood and Xray chest was normal.

Sixth day temperature $99.7 \mathrm{~F}$ pulse $111 \mathrm{bpm}$. CBC and CRP done. Seventh day Coughing started temperature $101 \mathrm{~F}$, pulse $118 \mathrm{bpm}$. I changed antibiotics to Amoxyclav and Levoflox. CRP was 12. Eight day temperature 100F Pulse 103 bpm. Ninth day temperature same 100F. 11th day CBC CRP and D dimer done except CRP (99) others were normal. 13th day Zifi started and other antibiotics omitted and CT chest advised and CT chest reported as Viral pneumonia mostly in both lower zone Corad grade 5/25. Steroid added on 13th day with tapering dose, 15 th day CRP was 23. Fever become normal, 18 th day CRP was 12 . And on 22 day it is below normal. During above period on examination Respiratory and Cardiosystem was normal.

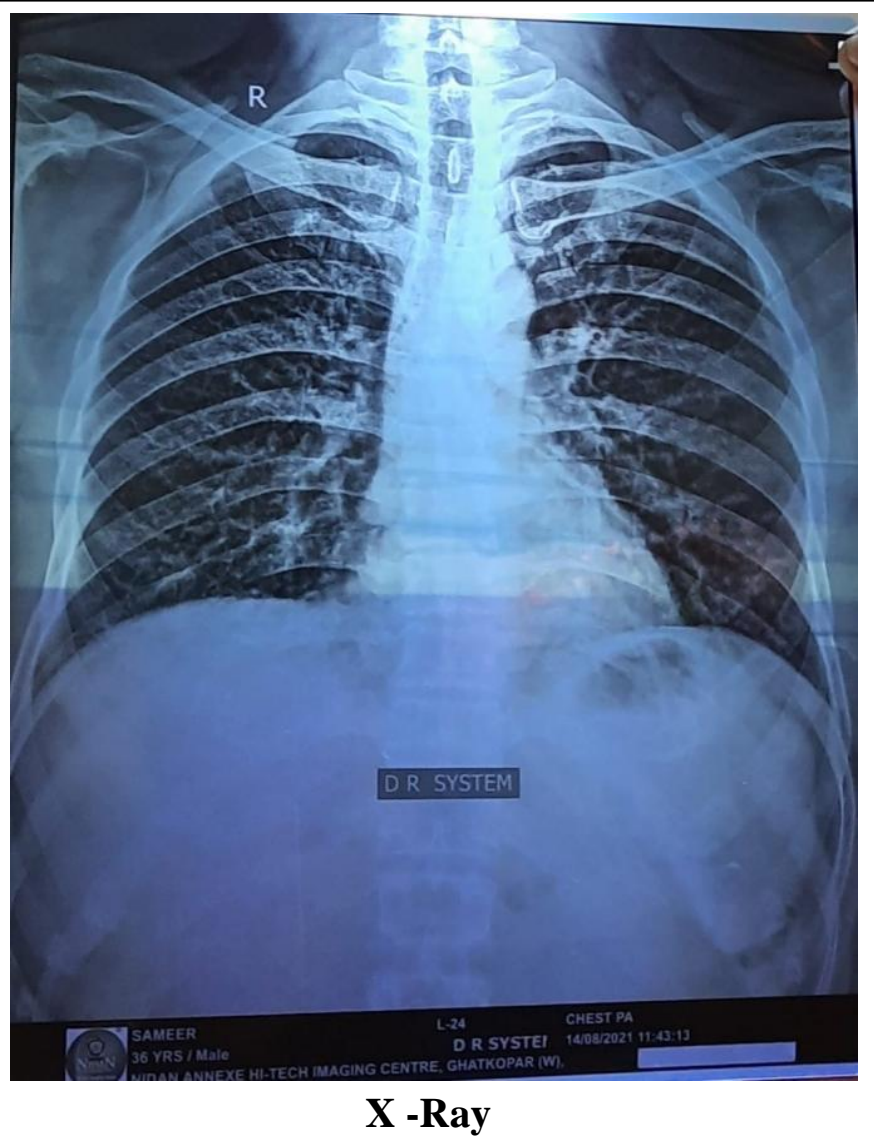

\section{Conclusion}

Covid-19 is characterized by marked variability in symptoms and any patient presenting with flu like symptoms with or without respiratory distress should be managed as Covid-19 until proven otherwise.

Detection of SARS-CoV-2 RNA through RT-PCR is the current gold standard for the diagnosis of COVID-19. However due to false negativity of RT-PCR many a times clinician has to rely on radiological investigations for the diagnosis of COVID-19. RT-PCR is confirmatory or specific for diagnosis but it is not sensitive and HRCT chest is the most sensitive but costly,

Computed tomography chest is the current gold standard for the imaging COVID-19 lung. CT scan is highly sensitive in detecting pulmonary changes and in some studies CT chest was found to be the far more sensitive than RT-PCR.4 Though not in guidelines whenever facility exist CT imaging should be performed and subsequent follow-u ps can be made through chest X-rays. ${ }^{(5)}$

Routine laboratory investigations are usually nonspecific. WBC is usually normal or low. There 
may be lymphopenia; a lymphocyte count $<1000$ has been associated with severe disease. The platelet count is usually normal or mildly low. The CRP and ESR are generally elevated but procalcitonin levels are usually normal. A high procalcitonin level may indicate a bacterial coinfection. The ALT/AST, prothrombin time, creatinine, D-dimer, CPK and LDH may be elevated and high levels are associated with severe disease. ${ }^{(6)}$

Early in the clinical course, the disease is primarily driven by the replication of SARS-CoV2. Later in the clinical course, the disease appears to be driven by a dysregulated immune/ inflammatory response to SARS-CoV-2 that leads to tissue damage. Based on this understanding, it is anticipated that therapies that directly target SARS-CoV-2 would have the greatest effect early in the course of the disease, while immunosuppressive/anti-inflammatory therapies are likely to be more beneficial in the later stages of COVID-19. ${ }^{(7)}$

Steroid was added on 13th as coronavirus replication stops after 9 days. There are 3 stages in Covid-19 Incubation period 3-5 days, Symptomatic period 7- 10days and Pulmonary period Early and late 9- 21 days. Steroid to be added in late phase of Pulmonary period in patient who is having moderate to severe disease. CT shows Viral Pneumonia, Gradual increase of CRP, persistent fever and H/o Hypertension goes in favor of Moderate to Severe disease.

Key Words: Covid-19, CRP, CT Chest,

\section{References}

1. Johns Hopkins University. Coronavirus Resource Center. October 9, 2020. Johns Hopkins University. https://coronavirus.jhu.edu/map.html. Accessed October 9, 2020.

2. Timeline: WHO's COVID 19 response. Available from: https://www.who/Int/emergencies/diseases /novel-coronavirus-2019/interactivetimeline
gclid=CjwKCAjwtNf6BRAwEiwAkt6UQi

ZuBpj903-1KLgvpAZA5zowCSie

bXrZTIOpD2Efe3nWzsBUuFKFnhoCfM YQAvD_BwE\#event-115.

3. Breaking Down Covid-19. A Living Textbook: Carey Kriz, Naiyer Imam, Sarah Zaidi.

4. https://www.covid19treatmentguidelines.n ih.gov/ on $9 / 1 / 2021$

5. Use of chest imaging in Covid-19. Available from; http;//www.who.int/publication/i/item/useof-chest-imaging-in-covid-19.

6. Breaking Down Covid-19. A Living Textbook: Carey Kriz, Naiyer Imam, Sarah Zaidi.

7. Therapeutic Management of Non hospitalized Adults With COVID-19 https://www.covid19treatmentguidelines.n ih.gov/ on 9/1/2021. 